\title{
Underwater Wireless Video Transmission for Supervisory Control and Inspection using Acoustic OFDM
}

\author{
Jordi Ribas \\ Massachusetts Institute of Technology \\ Cambridge, Massachusetts 02139 \\ Email: ribas@mit.edu
}

\author{
Daniel Sura \\ Massachusetts Institute of Technology \\ Cambridge, Massachusetts 02139 \\ Email: dsurfer@mit.edu
}

\author{
Milica Stojanovic \\ Northeastern University \\ Boston, Massachusetts 02115 \\ Email: millitsa@ece.neu.edu
}

\begin{abstract}
We study the feasibility of video transmission using an acoustic system for deep-sea oilfield supervisory control and inspection. Such a system is of interest over short distance links, with short delays and high available bandwidth, as near realtime imagery can be transmitted to monitor targeted underwater spots. This work includes three parts: (1) noise characterization for deep-sea oilfield environments, (2) video compression to reduce the amount of data to be transmitted through the channel, and (3) OFDM modulation to achieve high bit rates over the underwater link. Experimental testing was conducted at the MIT Sailing Pavilion premises, in the Charles River, Boston. The results demonstrate the usefulness of the coupled MPEG-4 compression and OFDM modulation, reaching bit rates on the order of $90 \mathrm{kbps}$ in a $115 \mathrm{kHz}$ acoustic band over a 200-meter link under a variety of channel conditions.
\end{abstract}

\section{INTRODUCTION}

The need for wireless image and video transmission underwater exists in many applications, such as port and harbor inspection, ecological monitoring, aqua-culture and fishing, subsea oil and gas equipment inspection and homeland security. Early efforts were made by Japanese scientists, who succeeded in transmitting a digital video showing a crab crawling at a depth of $1,000 \mathrm{~m}$ in an ocean trench [1]. This video was in fact a sequence of independent images, played at about one image every 10 seconds (enough to demonstrate the technology of the day). The frame rate of only a few frames every 10 seconds is not sufficient to capture an arbitrary video, but it may be quite sufficient to capture a remote ocean-floor scene such as that of a slowly crawling animal.

Since the time when these experiments were reported (more than a decade ago) progress has been made on both fronts: video compression, which enables transmission of full-resolution video at a much lower bit rate, and acoustic communications and signal processing, which enable the transmission of digital signals at a much higher bit rate and through channels that are much more challenging than a simple stable vertical link. Existing techniques for underwater

This work was supported in part by the MIT Sea Grant College Program under NOAA Grant Number NA06OAR4170019, MIT SG Project Number 2009-R/RCM-26, the Chevron MIT Energy Initiative program, project titled "Acoustic Communications for Ultra-Deepwater Oilfield Applications", 20082010, and ONR grants N00014-10-1-0576 and N00014-07-1-0202. acoustic transmissions and video compression algorithms are at this time mature enough to face the challenges of the data rate needed for real-time video transmission underwater.

The fact that underwater images and video are of low contrast enables them to be compressed to bit rates below those required for usual terrestrial images and video. This speaks in favor of the possibility to use readily available methods for video compression below $64 \mathrm{kbps}$, while advanced communication techniques provide the possibility to transmit at such bit rates over the acoustic channel. Specifically, video compression methods such as the standard MPEG-4 technique [2] or custom-designed wavelet-based methods [3], [4] can be used to compress the video, while recently developed algorithms for Orthogonal Frequency Division Multiplexing (OFDM) signal modulation and detection [5], [6] can be applied to efficiently use the limited acoustic bandwidth.

The purpose of the present research is to select and evaluate appropriate techniques for video compression and digital signal modulation and develop a system that is able to perform transmission and detection of signals over short distance links. A particular application of interest to our work is supervisory control of an Autonomous Underwater Vehicle (AUV) deployed in a deep-sea oilfield environment for purposes of monitoring and inspection. We envision a scenario where a vehicle is a short distance away from the object of interest, e.g. on a pipe, which in turn is linked to the shore operator via a cable. A video recorded by a camera onboard the vehicle is transmitted acoustically to the nearby receiver, which passes the signal on to the operator. The operator in turn issues a command signal to the vehicle, thus closing the control loop.

A particular feature of such a short range acoustic link is the noise that exists in a deep-sea oil drilling environment. The first task of our study was to assess the statistical properties of this noise. Ambient recordings were made in the Gulf of Mexico, and used to assess the spectral properties of the noise. These results indicated a suitable frequency range for acoustic communication between about $40 \mathrm{kHz}$ and $155 \mathrm{kHz}$. The video compression and acoustic communication system was then implemented in software, and local field tests were conducted in the chosen frequency band. Site-specific noise 

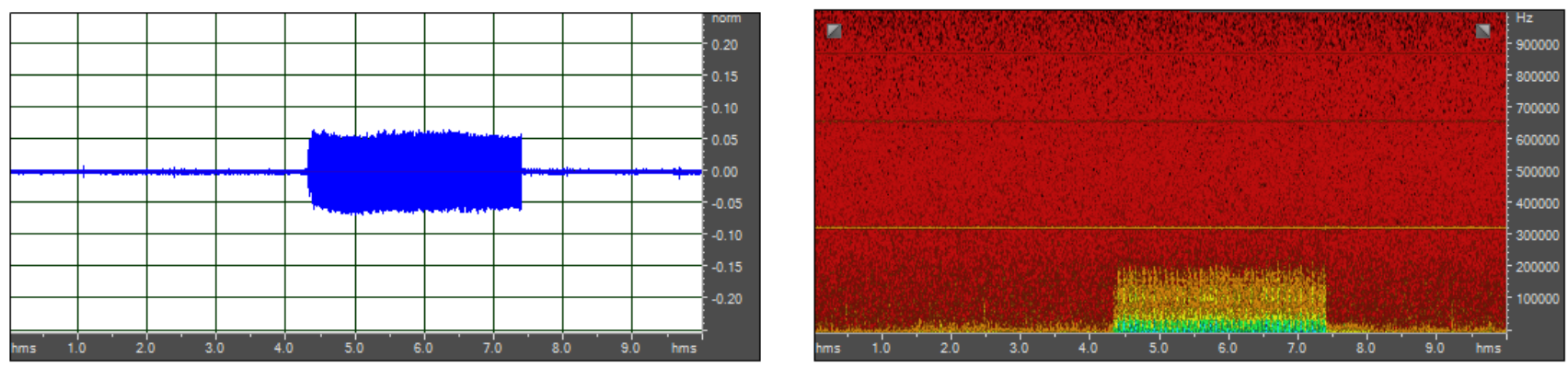

Fig. 1. Broadband buzzing noise captured in the Gulf of Mexico trials. The plot on the left shows the noise source in the time domain, while the plot on the right shows the broadband effects in the frequency domain.

of the deep sea was added externally to the recorded signals. Finally, the so-obtained signals were processed off-line, to assess the system performance with varying compression and communication techniques. Our results indicate that MPEG-4 compression and acoustic OFDM transmission offer a viable approach for video transmission at bit rates below $64 \mathrm{kbps}$ over distances on the order of $100 \mathrm{~m}$.

The paper is organized as follows. In Sec. II the noise analysis is presented. Video compression and signal modulation are described in Sec. III and IV. The system implementation is outlined in Sec. V. Sec. VI describes the preliminary simulation tests, while the results of field tests are presented in Sec. VII. Finally, we conclude in Sec. VIII.

\section{NOISE CHARACTERIZATION}

Typical oilfield environments have multiple noise sources, which include drilling and exploratory operations, underwater vehicle activity, and nearby communication systems such as dynamic positioning for surface ships. To assess the feasibility of using an acoustic OFDM system in an oilfield environment, a noise characterization study was performed via extensive measurements made in the Gulf of Mexico as part of a joint MIT/Chevron research project.

Ambient recordings were performed aboard Chevron's Discoverer Deep Seas drilling ship. This particular site was an exploratory well where a riser from the drilling ship was connected to a Blow Out Preventer (BOP) at the seafloor at a depth of about 6,200 ft. Several types of recognizable and unrecognizable noise sources were captured and identified. Some of these include noises from drilling activity, ROV operation, communication pings from the ship's dynamic positioning system, and several unknown types of buzzing and knocking noises. Fig. 1 shows one of the more peculiar noises captured, which had a buzzing sound and presents problems for acoustic communications due to its broadband nature. The plot on the left shows the noise source in the time domain with a duration of about 3.5 seconds, while the plot on the right shows the frequency domain content of the signal. Notice that the effects are quite broadband and could certainly interfere with an acoustic communication signal depending on the power and bandwidths used.

The next part of the analysis involved taking all of the noise sources and performing Power Spectral Density (PSD) analysis to determine suitable configurations for acoustic OFDM transmission. Fig. 2 shows a PSD summary containing all of the major types of noise sources captured during the deployments. Notice that the ROV activity presents the highest levels and quite broadband effects, followed by the peculiar buzzing noise previously mentioned.

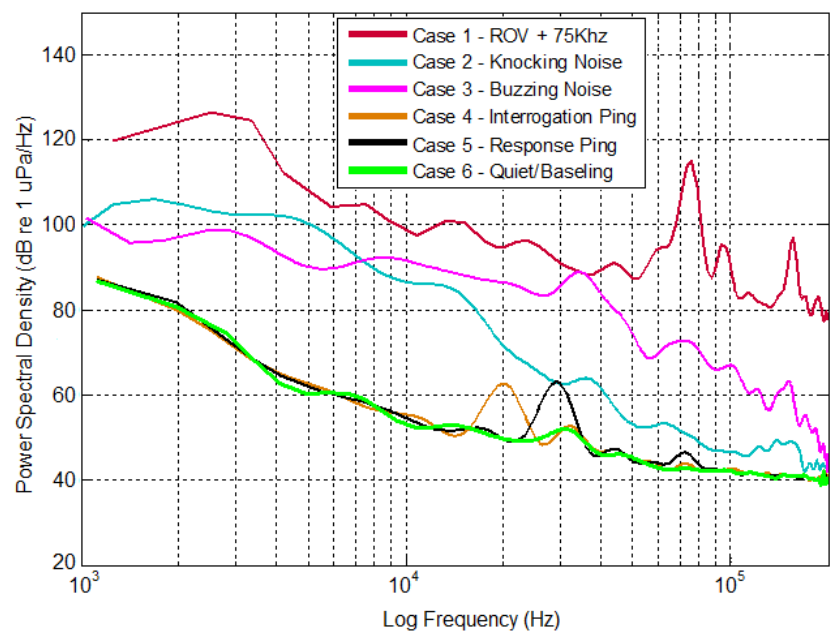

Fig. 2. Power spectral density estimates for noise sources captured in the Gulf of Mexico.

The PSD estimates were integrated over frequency bands of interest in order to obtain noise power levels over a $70 \mathrm{kHz}$ bandwidth. An OFDM transmission signal level was designed to operate under the presence of these noises with a $20 \mathrm{~dB}$ Signal to Noise Ratio (SNR) over the highest noise case (the peculiar buzzing noise). A simulation was performed using the captured buzzing recording and the OFDM transmission signal with a separation distance of $100 \mathrm{~m}$ between the transmitter and receiver. Fig. 3 shows the simulated signal captured on the receiver side, which contains a combination of the broadband buzzing and the OFDM communication signal centered at 120 $\mathrm{kHz}$. Notice that the OFDM signal blocks show up clearly in the plot on the right in the frequency domain. The OFDM signal is harder to identify in the time-domain plot, shown on the left, due to the broadband buzzing noise signature. 

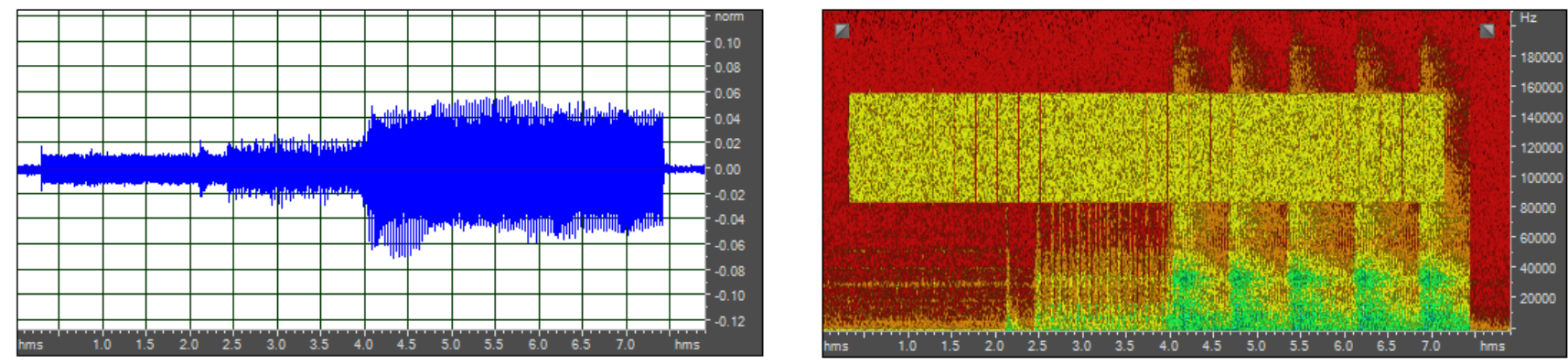

Fig. 3. Broadband buzzing noise source over generated OFDM signal. Plot on the left shows the time domain signal, and the plot on the right shows the frequency domain signal.

\section{VIDEO COMPRESSION}

Today, compression methods exist that can be used to transmit video at $64 \mathrm{kbps}$. In particular, the commerciallyavailable MPEG-4 encoders provide compression at this rate, by combining spatial and temporal techniques. Existing efforts at applying MPEG-4 compression for acoustic underwater video transmission include the ones in [7], where a singlecarrier modulation scheme operates over a short range (300 m). Wavelet-based algorithms have also been designed that deal with motion estimation and compensation for video applications [3]. Our study focuses on both the MPEG-4 and the wavelet-based techniques with target bit rates of $64 \mathrm{kbps}$ or less.

\section{A. MPEG-4 technique}

In the MPEG-4 standard, besides content-based functionalities for modern interactive multimedia systems, there are specific functionalities for very low bit rate video in error prone environments. The Very Low Bit-rate Video (VLBV) core provides algorithms and tools for applications operating at bit rates typically between 5 and 64 kbps. They support image sequences with low spatial resolution (typically up to Common Intermediate Format, CIF) and low frame rates (typically up to $15 \mathrm{~Hz}$ ). The specific functionalities for basic applications supported by the VLBV core include coding of conventional rectangular-shaped image sequences with high coding efficiency and high error robustness/resilience, low latency and low complexity for real-time multimedia communication applications.

A hybrid temporal-spatial coder (basic encoding scheme shown in Fig. 4) for natural and synthetic data coding has been used, with improved temporal random access and coding efficiency, and high robustness in challenging environments. The selected implementation calculates frame statistics during the video encoding process, and according to several parameters, the behavior of the algorithm is adjusted to meet the target bit rate requirement. These parameters must be selected in accordance with the target performance and the input file characteristics (resolution, frame rate, etc.). The following settings are defined: the reaction setting, which determines how slowly the encoder is allowed to react in deviation from target bit rate vs. actual bit rate; the averaging setting, which

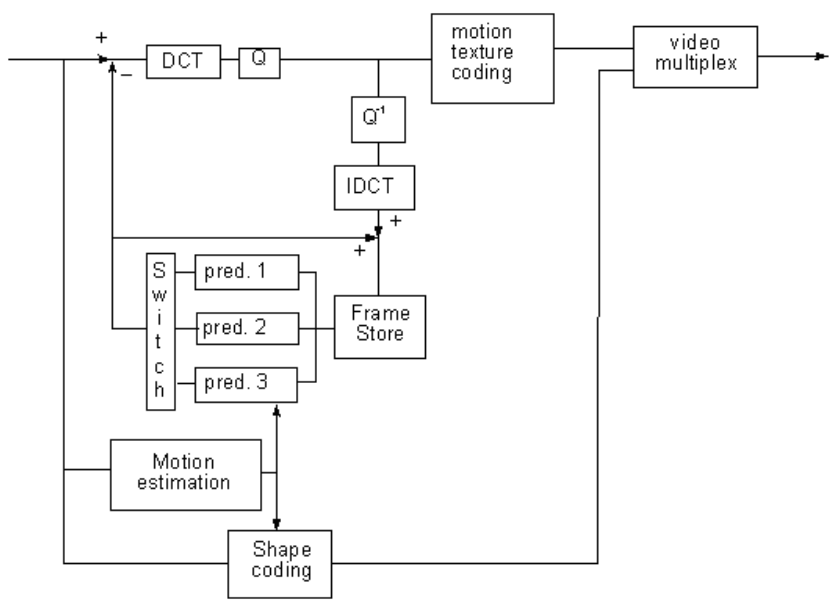

Fig. 4. MPEG-4 encoding scheme.
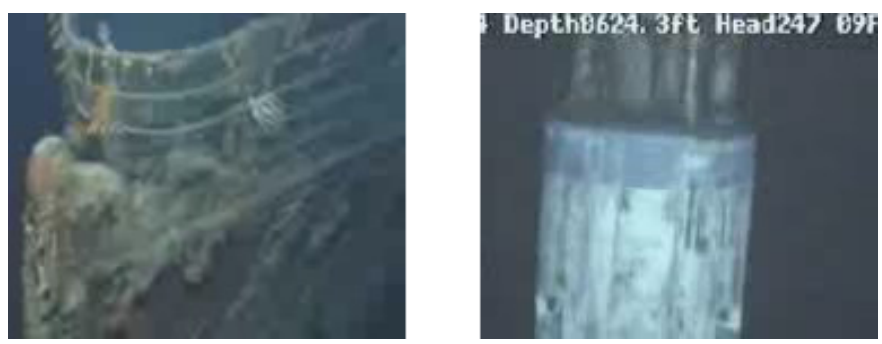

Fig. 5. Sample compressed frames with the MPEG-4 codec. Both images have Quarter CIF (QCIF) resolution $(176 \times 144$ pixels, scaled in the figure), $30 \mathrm{fps}$ frame rate and $64 \mathrm{kbps}$ encoding target bit rate.

indicates the number of frames for the sliding window used to gather statistics, and the smoother coefficient, which allows to adjust the possible deviation in the bit distribution along the frames of the sliding window.

Two raw files have been selected and coded in order to evaluate the performance of the MPEG-4 technique (samples from the resulting decoded video files are provided in Fig. 5). These encoded files also serve as input bits for the modulation process performed during the field experiments. For these two cases, the configurable internal parameters have been adjusted to provide maximum quality compressed footage for the selected videos. 


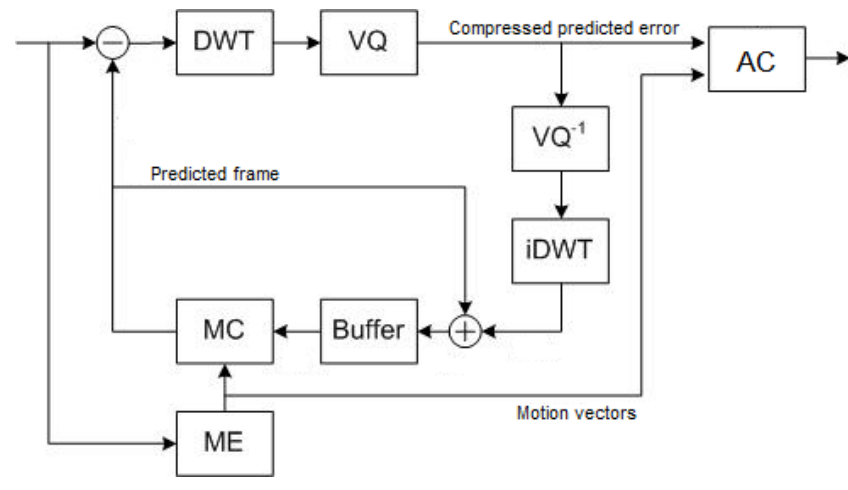

Fig. 6. Wavelet-based encoding scheme.
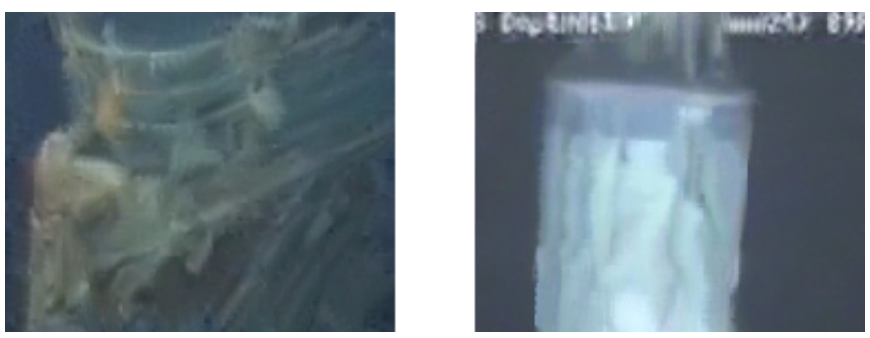

Fig. 7. Sample compressed frames with the wavelet-based codec. Both images have QCIF resolution $(176 \times 144$ pixels, scaled in the figure), $30 \mathrm{fps}$ frame rate and $64 \mathrm{kbps}$ encoding target bit rate.

\section{B. Wavelet-based technique}

The wavelet-based encoder algorithm includes techniques that deal with spatial and temporal redundancies in video sequences. In contrast to MPEG-4 compression, spatial redundancies are exploited by applying the Discrete Wavelet Transform (DWT) to each of the frames composing the video sequence. A motion compensation algorithm [3] to reduce temporal redundancies has been incorporated, which is a core functionality of the MPEG-4 encoder.

The codec is based on the popular Differential Pulse Code Modulation (DPCM) model, which is widely used in video compression standards (see Fig. 6). Its main components are the DWT multilevel decomposition of the input frame using the bi-orthogonal wavelet, quantization of the DWT coefficients using Vector Quantization (VQ) with 40 different codebooks and coding rates from $1 / 32$ to 4 bits/sample, blockbased Motion Estimation and Compensation (ME, MC), and adaptive Arithmetic Coding (AC) of quantized coefficients.

The same raw files used for the MPEG-4 tests have been coded with the wavelet-based method. Sample compressed frames are shown in Fig. 7. The images are smoother due to the high quantization ratio for the high subbands of the wavelet decomposition and, as expected, no blocking effect is noticed at all.

\section{OFDM MODULATION}

Multicarrier modulation based on OFDM was used for acoustic transmission. Effective detection methods that were developed over the last years and showed excellent performance at low computational cost were used [5], [8].

\section{A. Transmitter}

Typical zero-padded OFDM modulation was used with $K$ carriers, where the input data is received from either the wavelet-based encoder or the MPEG-4 encoder once the recorded video clip has been compressed. Channel coding is performed on the compressed bit stream, followed by serialto-parallel conversion, mapping into symbols and interleaving. The so-obtained $K$ symbol streams, $d_{k}(n), k=0, \ldots, K-1$ form the signal

$$
u(t)=\sum_{n} \sum_{k=0}^{K-1} d_{k}(n) e^{j 2 \pi k \Delta f\left(t-n T^{\prime}\right)} g\left(t-n T^{\prime}\right)
$$

where $g(t)$ is a rectangular pulse in time with unit amplitude and duration $T ; T^{\prime}=T+T_{g}$, where $T_{g}$ is the guard interval, which is longer than the multipath spread; and $\Delta f=1 / T$ is the carrier spacing.

The modulated, passband signal is then obtained as

$$
s(t)=\operatorname{Re}\left\{u(t) e^{j 2 \pi f_{0} t}\right\}
$$

where $f_{0}$ is the lowest subcarrier frequency, and $f_{k}=$ $f_{0}+k \Delta f$ is the $k$-th subcarrier frequency. The symbol rate is $R=K /\left(T+T_{g}\right)$ symbols per second (sps), and the signal bandwidth is defined as $B=K \Delta f$. The resulting bandwidth efficiency is given by $R / B=1 /\left(1+T_{g} / T\right) \mathrm{sps} / \mathrm{Hz}$.

Transmission is organized in frames. Each frame, consisting of a number of OFDM blocks, is preceded by a high-resolution broadband synchronization preamble. The system block diagram is shown in Fig. 8 .

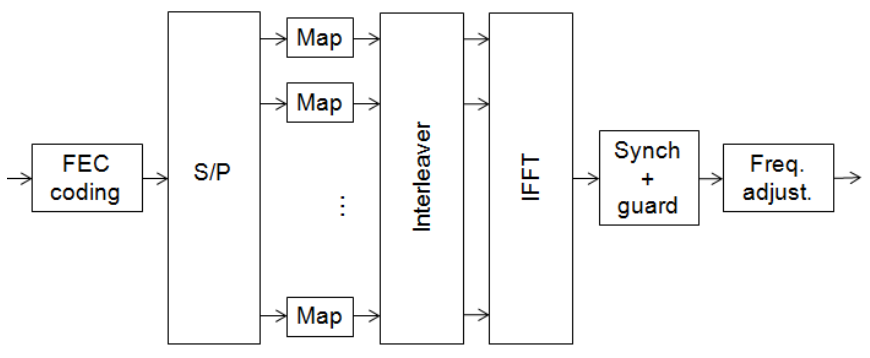

Fig. 8. Transmitter.

Several Peak to Average Ratio (PAR) reduction techniques have also been implemented. These techniques include clipping and filtering [9], symbol interleaving [10], in-band tone reservation [11], and out-of-band tone insertion [12].

\section{B. Receiver}

The receiving block diagram is illustrated in Fig. 9. Frontend band-pass FIR filtering is used to suppress the out-of-band noise. Frame synchronization is then performed via preamble correlation. The OFDM blocks are then down-converted, and 
the FFT demodulation is applied. The resulting signal is modeled as

$$
y_{k}(n)=H_{k}(n) d_{k}(n) e^{j \theta_{k}(n)}+z_{k}(n)
$$

where $H_{k}(n)$ represents the channel coefficient (transfer function evaluated at the $k$-th carrier), $\theta_{k}(n)$ represents the phase shift due to the motion-induced Doppler effect, and $z_{k}(n)$ is the residual in-band noise. The post-FFT processing includes data detection and decoding, deinterleaving, and parallel-to-serial conversion. The so-obtained bits are finally used to reconstruct the video.

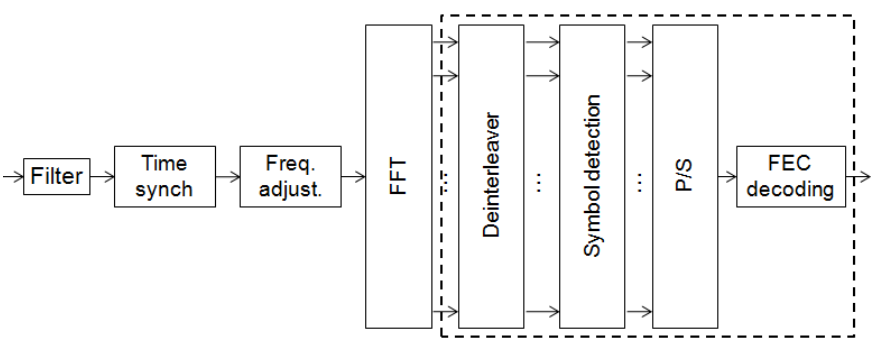

Fig. 9. Receiver scheme.

Two types of detection algorithms have been implemented: differentially coherent detection and coherent detection.

1) Differentially coherent detector: Differentially coherent detection is a simple and efficient method for use with OFDM signals. We focus on differential detection in frequency, as it goes hand in hand with a bandwidth-efficient design of an OFDM system. Unlike coherent detection, which relies on explicit phase and channel estimation, differentially coherent detection extracts this information implicitly, relying on the frequency coherence between adjacent subbands.

Differentially coherent detection requires differential encoding of the transmitted data stream. If we denote by $b_{k}(n)$ the original data stream, differential encoding will yield

$$
d_{k}(n)=d_{k-1}(n) b_{k}(n), \quad k=0, \ldots, K-1
$$

The encoding process begins with $d_{-1}(n)=1$.

Differentially coherent detection relies on the assumption that $H_{k}(n) \approx H_{k-1}(n)$ and $\theta_{k}(n) \approx \theta_{k-1}(n)$, and generates the estimate of the original data symbol as

$$
\hat{b}_{k}(n)=\frac{y_{k}(n)}{y_{k-1}(n)}
$$

2) Coherent detector: Coherent detection was implemented using the algorithm [5]. This algorithm focuses on postFFT processing using adaptive channel estimation and nonuniform Doppler compensation. Unlike differentially coherent detection, this algorithm relies on temporal coherence between adjacent OFDM blocks. It involves four steps: preliminary channel and phase compensation using estimates from the previous block (namely $\hat{H}_{k}(n-1), \hat{\theta}_{k}(n-1)$ ), estimation of the Doppler scaling factor using tentative symbol decisions, coding-oriented symbol detection, and channel estimation.
Channel estimation is performed in the impulse response domain, taking into account the possibility of channel sparseness [8].

\section{SYSTEM IMPLEMENTATION}

The transmitter block diagram is shown in Fig. 10.

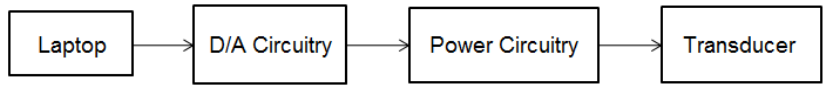

Fig. 10. Transmitter block diagram.

The transmitting side laptop is used to perform video compression and to generate the signal waveform for subsequent transmission. In the current implementation, raw video files are encoded using a $\mathrm{C}++$ software implementation of either MPEG-4 codec or wavelet-based technique, and the OFDM signal modulation/detection is performed in MATLAB. The result is stored in a wave-file. A custom script is executed to send the signal to the Digital to Analog (D/A) processing board.

The D/A circuitry utilizes a Universal Software Radio Peripheral (USRP) board, which has sampling capabilities sufficient for all of our acoustic applications.

The radio interface of the USRP is substituted by an acoustic one. The electrical signal produced on the USRP board is fed to a custom made Printed Circuit Board (PCB) which produces a tuned electrical signal at a power level needed to drive the acoustic transducer.

The transducer was custom designed for MIT by ITC transducers, specifically for deepwater omni-directional operation.

The receiver block diagram is shown in Fig. 11.

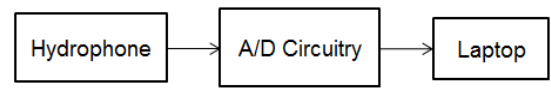

Fig. 11. Receiver block diagram.

A custom-designe hydrophone, provided by Reson specifically for deepwater operation, was used to capture the acoustic signal. A/D conversion is performed using another USRP board. The so-obtained signal is stored in wave-file format on the receiving laptop computer. Signal processing and video reconstruction are then performed off-line.

The operational frequency band is set to $40 \mathrm{kHz}-155$ $\mathrm{kHz}$ based on the noise characteristics. The selected OFDM configurations are summarized in Tab. I, and the candidate channel codes are summarized in Tab. II. An integer number of codewords are transmitted in each OFDM block, thus the channel utilization (given in percentage of the total number of subcarriers) depends on the $\mathrm{BCH}$ code.

\section{Vi. Preliminary evaluation}

Prior to field experiments, the system design was validated via simulation to determine the target Bit Error Rate (BER) for proper decoding of the video files. A simplified synthetic 


\begin{tabular}{|c|c|c|c|}
\hline \# subcarriers & carrier spacing & OFDM symbol duration & bandwidth efficiency \\
\hline$K$ & $\Delta f=B / K[\mathrm{~Hz}]$ & $T=1 / \Delta f[\mathrm{~ms}]$ & $R / B=T /\left(T+T_{g}\right)[\mathrm{sps} / \mathrm{Hz}]$ \\
\hline 8192 & 14.04 & 71.2 & 0.88 \\
\hline 16384 & 7.02 & 142.5 & 0.93 \\
\hline
\end{tabular}

TABLE I

OFDM SIGNAL PARAMETERS USED FOR THE EXPERIMENTS $(B=115 \mathrm{kHz}, \mathrm{Tg}=10 \mathrm{~ms})$.

\begin{tabular}{|c|c|c|c|c|c|c|c|c|c|}
\cline { 2 - 10 } \multicolumn{1}{c|}{} & \multicolumn{10}{c|}{ BCH coding } \\
\cline { 2 - 11 } \multicolumn{1}{c|}{} & $7 / 4$ & $15 / 11$ & $31 / 21$ & $63 / 51$ & $63 / 30$ & $63 / 18$ & $63 / 10$ & $127 / 120$ & $255 / 239$ \\
\hline Coding ratio & 1.75 & 1.36 & 1.48 & 1.24 & 2.10 & 3.50 & 6.30 & 1.06 & 1.07 \\
\hline Ch. utilization (\%) & 88 & 94 & 97 & 98 & 98 & 98 & 98 & 99 & 100 \\
\hline Correction (bits) & 1 & 1 & 2 & 2 & 6 & 10 & 13 & 1 & 2 \\
\hline
\end{tabular}

TABLE II

BCH CODES.

channel was used assuming a nominal geometry of a shallow water $200 \mathrm{~m}$ link, and actual deep-sea noise recordings were added to the simulated signal.

\section{A. Video detection}

A simple study was made by introducing random errors to the encoded video files and the video decoding performance was evaluated by analyzing the visual quality versus BER. Sample decoded frames with the MPEG-4 codec are shown in Fig. 12. The established target BER for acceptable performance was set below $10^{-3}$.
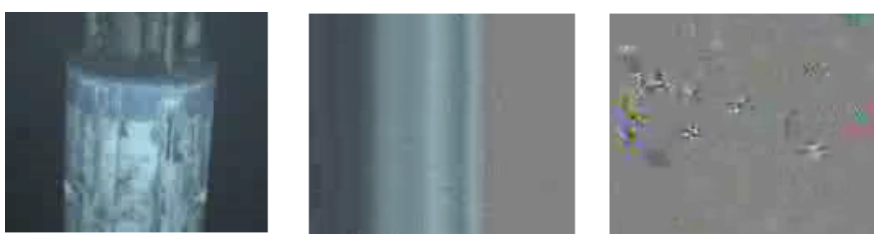

Fig. 12. Sample video detection with QCIF resolution $(176 \times 144$ pixels, scaled in the figure) and $30 \mathrm{fps}$ frame rate. $\mathrm{BER}=0 \%$ (left), $\mathrm{BER}=0.12 \%$ (center), and $\mathrm{BER}=1.17 \%$ (right)

\section{B. Channel simulation}

The block diagram of the channel simulator is shown in Fig. 13. Simulation includes the effects of multipath, attenuation over an underwater link, and addition of noise from recordings. For purposes of a simple design validation, the effects of time-variation are not included.

Three multipath arrivals were considered, at $0 \mathrm{msec}$ (direct path), $1 \mathrm{msec}$, and $2 \mathrm{msec}$, with different reflection coefficients (1, 0.6 and 0.3 , respectively).

Signal attenuation for a link of distance $l$ and frequency $f$ was evaluated using Thorp's formula [13]

$$
A(l, f)=l^{k} a(f)^{l}
$$

where the spreading factor was set to $k=1.5$.

Different noise recordings were added to the signal, and detection results showed good quality video reconstruction

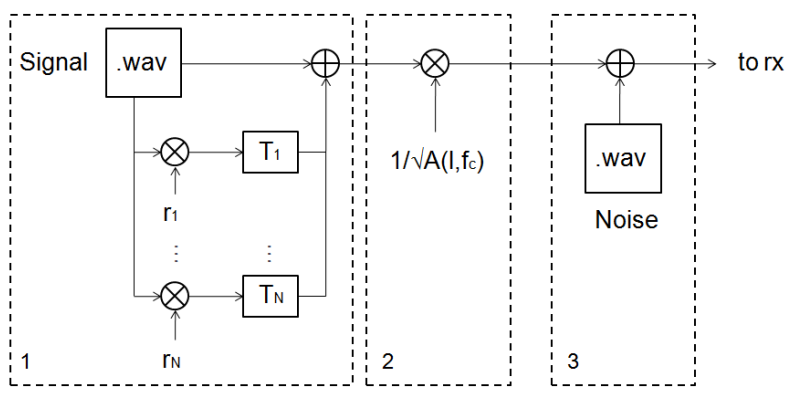

Fig. 13. Channel simulator block diagram.

for most cases. The performance worsened with more severe noise examples, but good performance was maintained for simulated distances below 500 meters. Sample Mean Square Error (MSE) of the detected constellations and coded BER results are illustrated in Fig. 14. The MSE remains below $-5 \mathrm{~dB}$, ensuring successful decoding, with no bit errors after decoding using the $\mathrm{BCH}(63,18)$ code.

\section{EXPERIMENTAL RESULTS}

Field experiments were conducted at the MIT Sailing Pavilion premises, with the transmitter located on the dock and the receiver on a boat, as illustrated in Fig. 15. The distance between the transmitter and the receiver was 200 meters, and the boat was positioned with the aid of a Global Positioning System (GPS) handheld. The boat was anchored to keep the distance fixed, and the transmitter and receiver were stabilized by weights to avoid vibrations and motion.

Three types of tests were conducted: channel coding tests, to determine the appropriate coding ratio to meet the performance requirements; OFDM modulation tests, to select the number of carriers and the most effective detection scheme; and consistency analysis, to check the performance reliability in varying conditions.

\section{A. Channel coding}

A data sequence of 16 OFDM blocks was transmitted over the 200-meter link using the codes of Tab. II, and the perfor- 

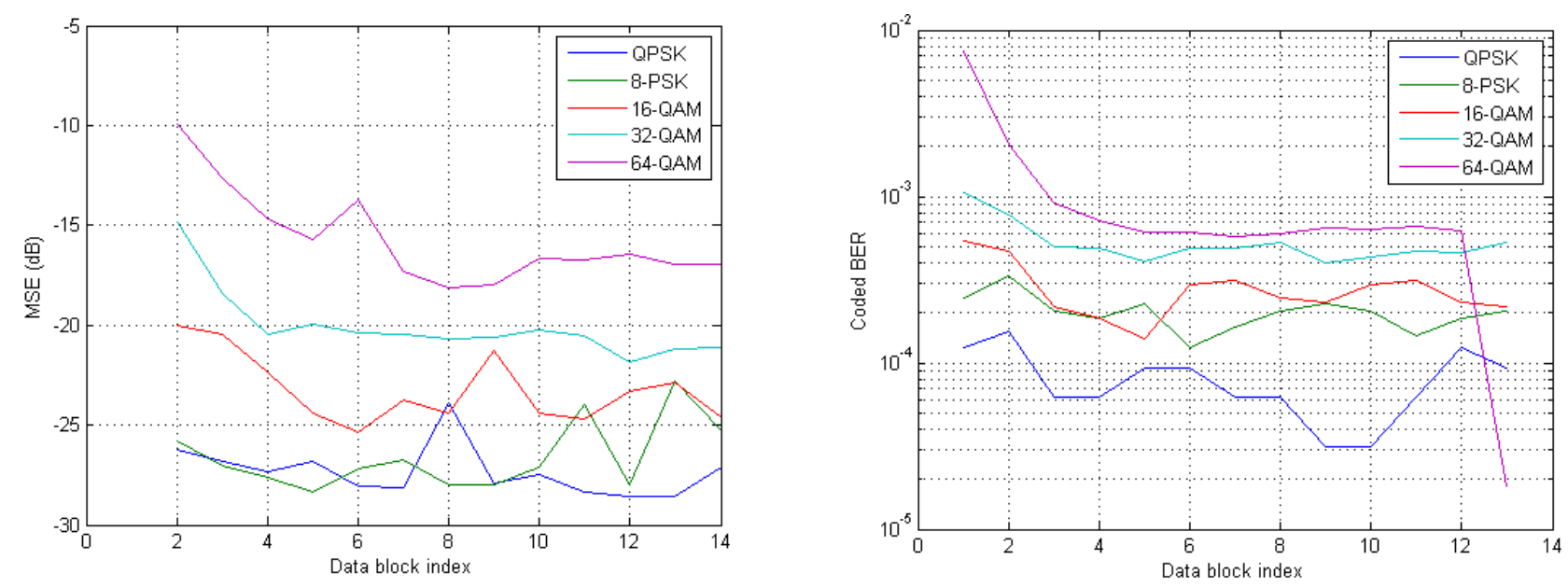

Fig. 14. Simulation test results. Sample MSE (left) and BER before decoding (right) for different subcarrier modulations. Parameters: 200-meter link, average noise, $\mathrm{BCH}(63,18)$ coding.

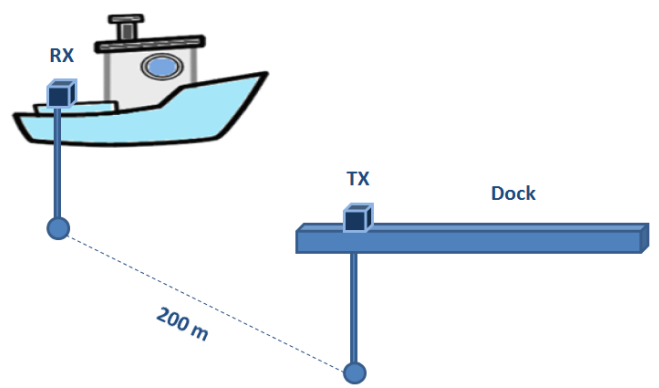

Fig. 15. Transmission deployment.

mance was assessed in terms of the BER after decoding. Fig. 16 summarizes the results obtained using coherent detection. The $\mathrm{BCH}(63,30)$ code clearly provides good performance, and so do the $\operatorname{BCH}(63,18)$ and $\operatorname{BCH}(63,10)$. In fact, the latter two codes consistently provided error-free performance (not shown in figure). Therefore, a coding rate of about $1 / 2$ is needed for consistent performance.

The BER increase with time (Fig. 16) occurs due to the channel estimation errors inherent to coherent detection. Channel estimation errors, caused by erroneous symbol decisions accumulate with time, causing a gradual loss of track and degrading the overall performance. This problem can in principle be alleviated through fine tuning of the receiver parameters and by reducing the OFDM block duration. An alternative approach is to use differentially coherent detection, which may be a preferred practical solution, as it does not require any parameters to be tuned. It also does not rely on block-toblock temporal coherence, but instead processes each block independently. As long as the block duration is within the time coherence of the channel, such that no inter-carrier interference is generated, differentially coherent detection capitalizes on longer block durations (narrower carrier spacing ensures more coherence between adjacent subbands). It thus supports a

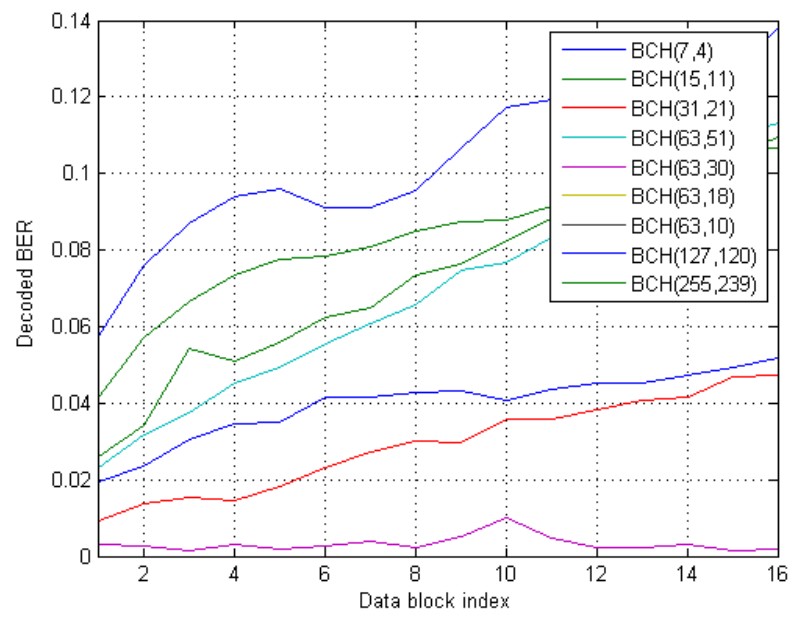

Fig. 16. Decoded sequence time BER for different BCH codes in a 200-meter link with 8-PSK subcarrier modulation.

simple and bandwidth-efficient system design.

\section{B. Subcarrier modulation}

Different subcarrier modulations were evaluated in light of bit rate and performance that they provided in the experiments. Sample constellations of the estimated data symbols in a 200meter link are shown in Fig. 17.

The BER performance is shown in Fig. 18. For most of the configurations, QPSK and 8-PSK provided satisfactory performance, while higher-level modulations (16-QAM, 32-QAM and 64-QAM) suffer an obvious loss. Sample reconstructed frames from the experiments are provided in Fig. 19 for 8PSK subcarrier modulation and MPEG-4 compression.

\section{Consistency}

Finally, a consistency study was performed for the 200meter experimental link. The 8-PSK modulation was selected, 

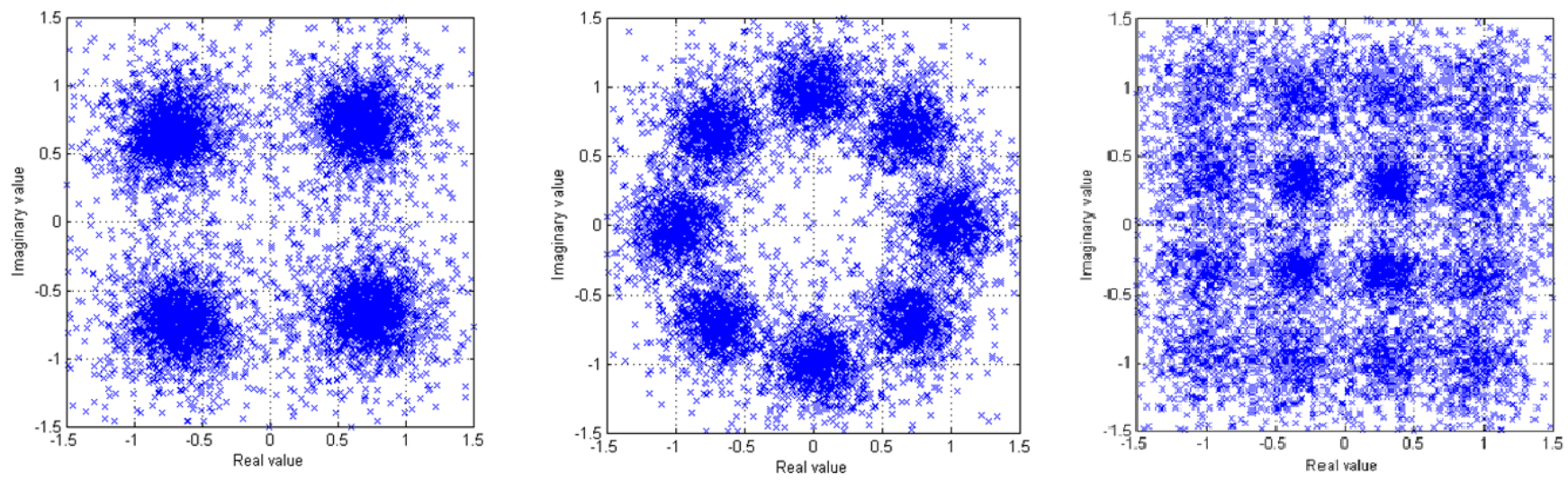

Fig. 17. QPSK (left), 8-PSK (center) and 16-QAM (right) constellations in a 200-meter link with BCH(63,18) coding.
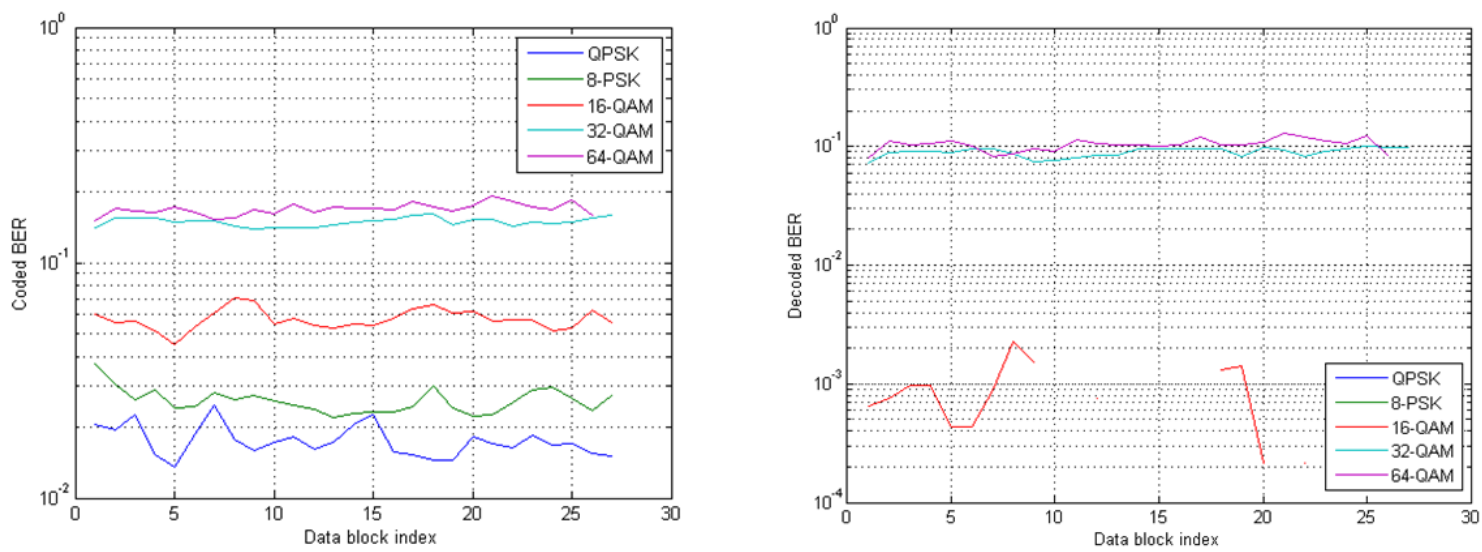

Fig. 18. BER before (left) and after decoding (right) for different subcarrier modulations in a 200-meter link with BCH(63,18) coding.
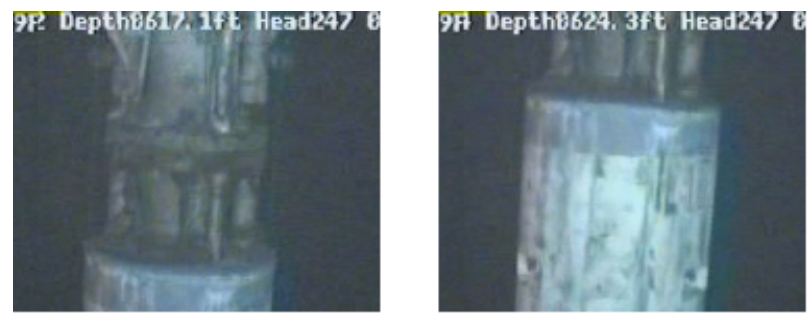

Fig. 19. Sample QCIF reconstructed frames (scaled in the figure) with MPEG-4 codec, 8-PSK subcarrier modulation and $\mathrm{BCH}(63,18)$ coding.

and two types of codes were used for comparison: $\mathrm{BCH}(63,18)$ and $\mathrm{BCH}(63,30)$. A number of consecutive transmissions lasting several seconds $(2.61$ seconds for the lighter code and 4.14 seconds for the heavier code) were made, separated by a one-second interval. The consistency test results are shown in Fig. 20, in terms of the MSE and the BER.

The $\operatorname{BCH}(63,18)$ code always met the BER requirements, while the $\operatorname{BCH}(63,30)$ code did not. The corresponding data rates for the candidate configurations are provided in Tab. III. We note that 8-PSK with $\mathrm{BCH}(63,18)$ provides a bit rate in excess of $64 \mathrm{kbps}$, as well as good performance in our field tests. Judging by these results, we conclude that near realtime video transmission over horizontal links on the order of a hundred meters is indeed a feasible task.

\begin{tabular}{|c|c|c|c|c|}
\hline \multirow{2}{*}{$K$} & \multirow{2}{*}{ Modulation } & \multicolumn{3}{|c|}{ BCH coding } \\
\cline { 3 - 5 } & & $63 / 30$ & $63 / 18$ & $63 / 10$ \\
\hline \multirow{2}{*}{8192} & QPSK & 94.54 & 56.73 & 31.51 \\
\cline { 2 - 5 } & 8 -PSK & 141.81 & 85.09 & 47.27 \\
\hline \multirow{2}{*}{16384} & QPSK & 100.74 & 60.45 & 33.58 \\
\cline { 2 - 5 } & $8-$-PSK & 151.11 & 90.67 & 50.37 \\
\hline
\end{tabular}

TABLE III

DATA BIT RATES (IN $k b p s$ ).

\section{CONCLUSIONS}

The feasibility of real-time video transmission over short horizontal acoustic links was addressed. The standard MPEG4 video compression technique and a wavelet-based method were combined with acoustic transmission based on coded OFDM modulation. Experimental results confirmed the possibility to achieve good quality signal detection (target BER less than $10^{-3}$ ) at bit rates around $64 \mathrm{kbps}$, the standard rate required by the MPEG-4 compression. 

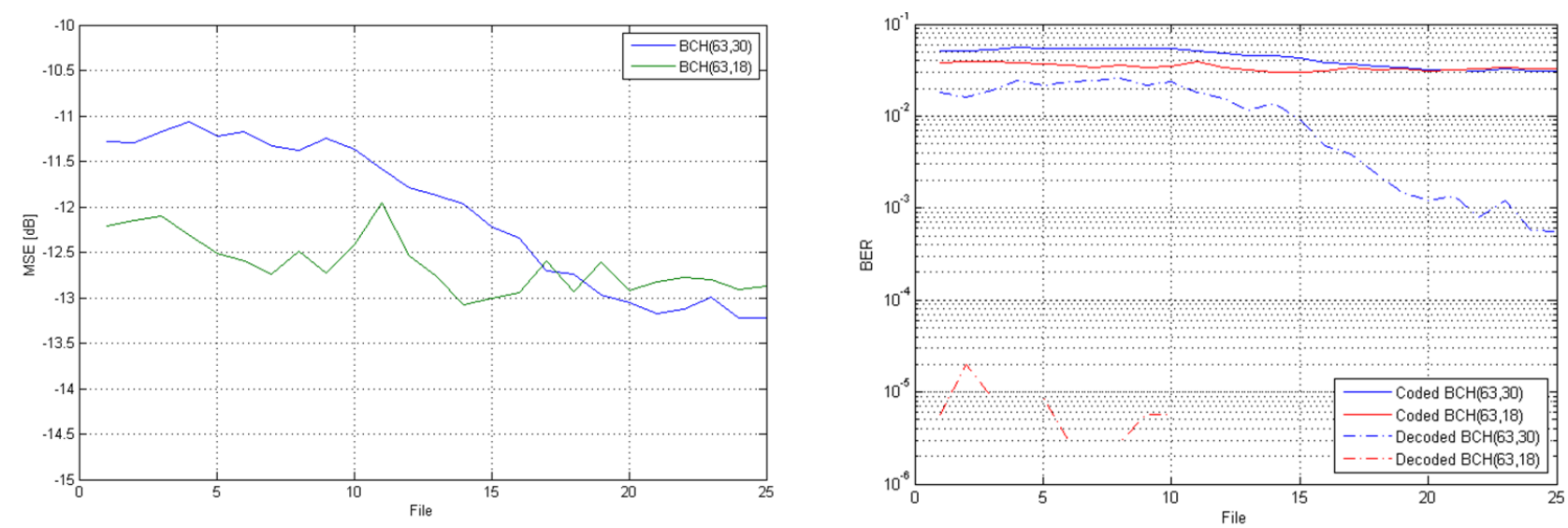

Fig. 20. Time MSE and time BER for 25 consecutive video transmissions with 8-PSK subcarrier modulation in a 200-meter link.

The experiments were designed so as to mimic our end goal of developing a communication system for supervisory control of an autonomous vehicle deployed in a deep-sea oil field for inspection and monitoring of the equipment. With limited access to deep sea, the tests were confined to a shallow water channel (which is possibly harsher than the deep-sea short link) with the addition of actual noise recorded in the deep waters in the Gulf of Mexico. A number of candidate modulation/detection and coding schemes were investigated. The frequency band was set to $40-155 \mathrm{kHz}$, which appears to offer a good region in terms of the relevant noise. Differentially coherent detection (with differential encoding across carriers) was found to offer consistency and robustness needed for longterm deployments, as well as simplicity of implementation. A selected suite of $\mathrm{BCH}$ codes indicated that a code rate of at most 1/2 may be sufficient for the application of interest. QPSK and 8-PSK modulation formats, together with a large number of carriers (8192 and higher) emerged as the best candidates for achieving the necessary bit rates.

Future work will focus on full software system implementation to enable on-line processing, as well as on testing in deep-sea environments.

\section{REFERENCES}

[1] M. Suzuki and T. Sasaki, "Digital acoustic image transmission system for deep sea research submersible," Proc. IEEE Oceans'92 Conference, September 1992.

[2] K. Jinzenji, H. Watanabe, S. Okada, and N. Kobayashi, "Very low bit rate video compression using MPEG-4 sprite coding," Proceedings of IEEE International Conference on Multimedia and Expo, August 2001.

[3] D. Hoag, V. Ingle, and R. Gaudette, "Low bit-rate coding of underwater video using wavelet-based compression algorithms," IEEE J. Oceanic Eng., vol. 22, pp. 393-400, April 1997.

[4] L. Qing-Zhong, W. Bing, W. Wen-Jin, and G. Xiao-Ling, "An efficient underwater video compression algorithm for underwater acoustic channel transmission," International Conference on Communications and Mobile Computing, January 2009.

[5] M. Stojanovic, "Low complexity OFDM detector for underwater acoustic channels," IEEE Oceans Conference, September 2006.

[6] K. Tu, D. Fertonani, T. M. Duman, and P. Hursky, "Mitigation of intercarrier interference in OFDM systems over underwater acoustic channels," Proceedings of IEEE Oceans Conference, May 2009.
[7] J. Kojima, T. Ura, H. Ando, and K. Asakawa, "High speed acoustic data link transmitting moving pictures for autonomous underwater vehicles," Proc. International Symposium on Underwater Technology, April 2002.

[8] M. Stojanovic, "OFDM for underwater acoustic communications: Adaptive synchronization and sparse channel estimation," Proc. International Conference on Acoustics, Speech, and Signal Processing (ICASSP'08), April 2008.

[9] X. Li and L. J. Cimini, "Effects of clipping and filtering on the performance of OFDM," Communications Letters, IEEE, vol. 2, no. 5 , May 1998.

[10] A. Jayalath and C. Tellambura, "Reducing the peak-to-average power ratio of orthogonal frequency division multiplexing signal through bit or symbol interleaving," Electronics Letters, vol. 36, no. 13, June 2000.

[11] B. Krongold and D. Jones, "An active-set approach for OFDM PAR reduction via tone reservation," Signal Processing, IEEE Transactions, vol. 52, no. 2, February 2004.

[12] G. Rojo and M. Stojanovic, "Peak-to-average power ratio (PAR) reduction for acoustic OFDM systems," Proc. IEEE Oceans'09 Conference, Biloxi, MS, October 2009.

[13] L.Berkhovskikh and Y.Lysanov, "Fundamentals of ocean acoustics," New York: Springer, 1982. 